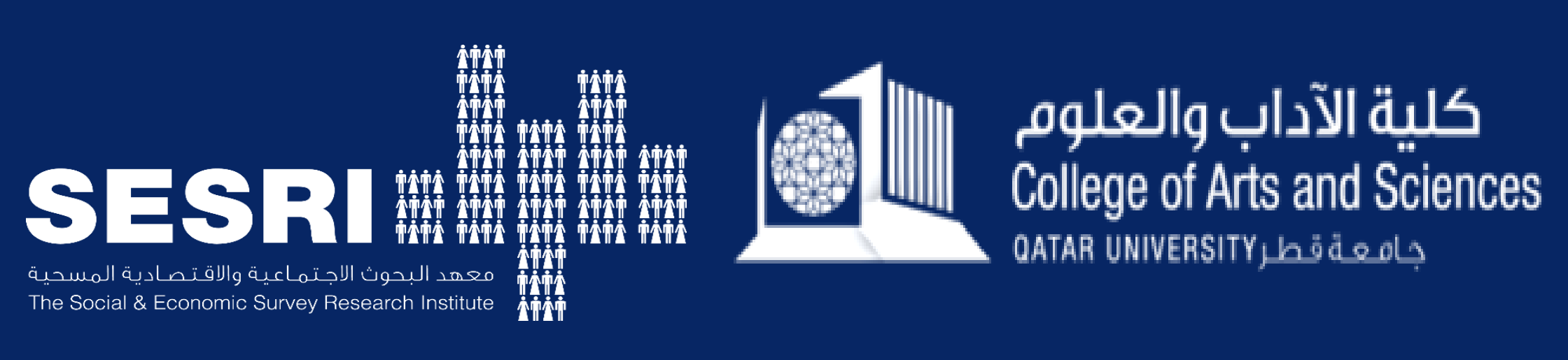

Faculty, Social Sciences and Humanities

\title{
Factors Associated with Depression 8 Anxiety in the Adult Population of Qatar after the first COVID-19 Wave : a Cross-sectional Survey
}

Salma Mawfek Khaled, Iman Amro, Lina Bader, Peter Woodruff, Majid A Alabdulla, Tarek Bellaj, Yousri Marzouk, Youssef Hasan, Ibrahim M Al-Kaabi \& Peter M Haddad

\section{Background}

- There is lack of data from Arabicspeaking countries on risk factors for depression and anxiety during the COVID-19 pandemic.

- Country-specific data are necessary given differences in culture, demographics and COVID-19 infection/ mortality rates.

- Data are also needed from different time points in the pandemic

\section{Aims}

To identify the factors associated with symptoms of depression-anxiety in the adult population of Qatar after the first COVID-19 wave.

\section{Methods}

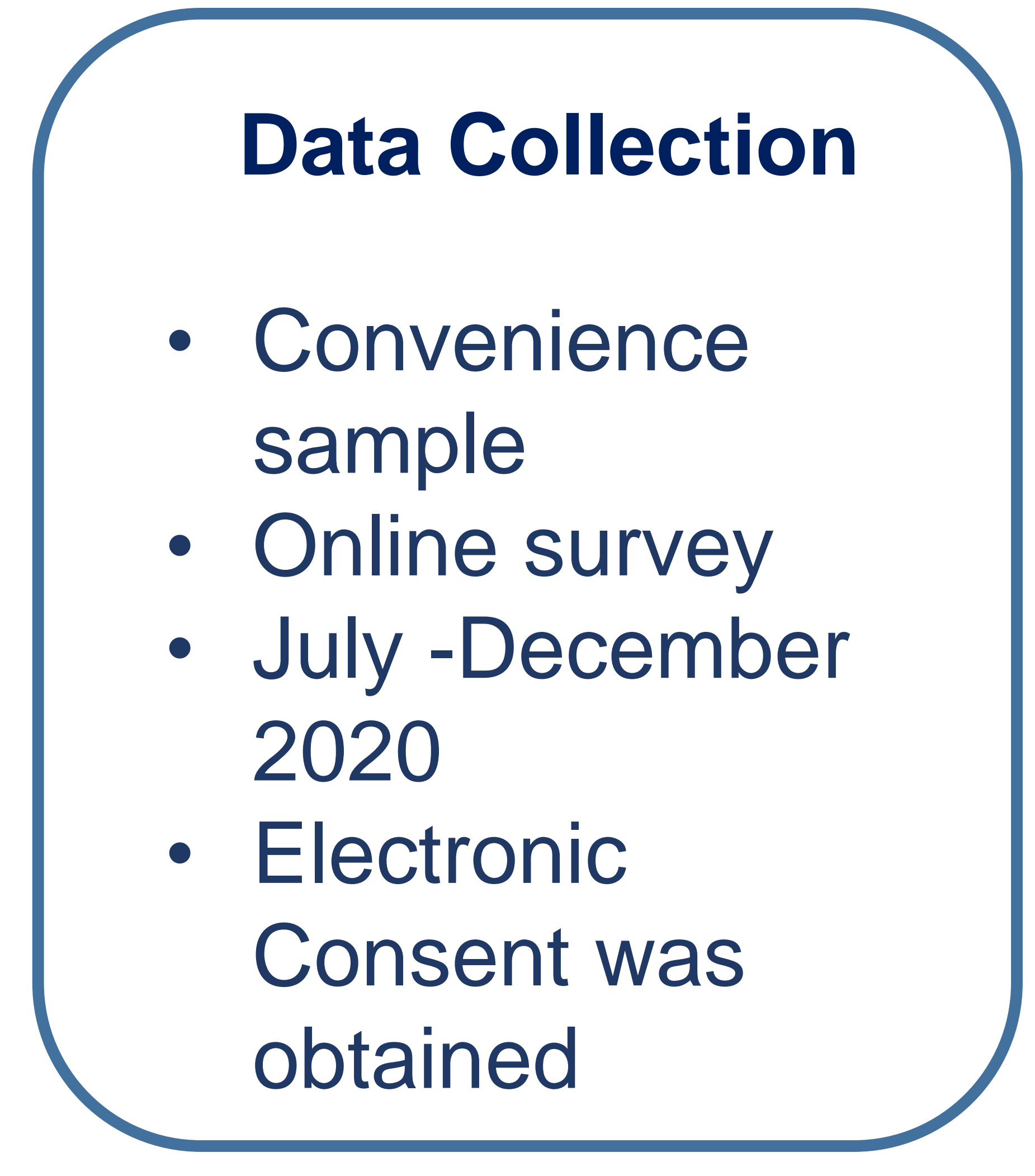

\section{Instruments}

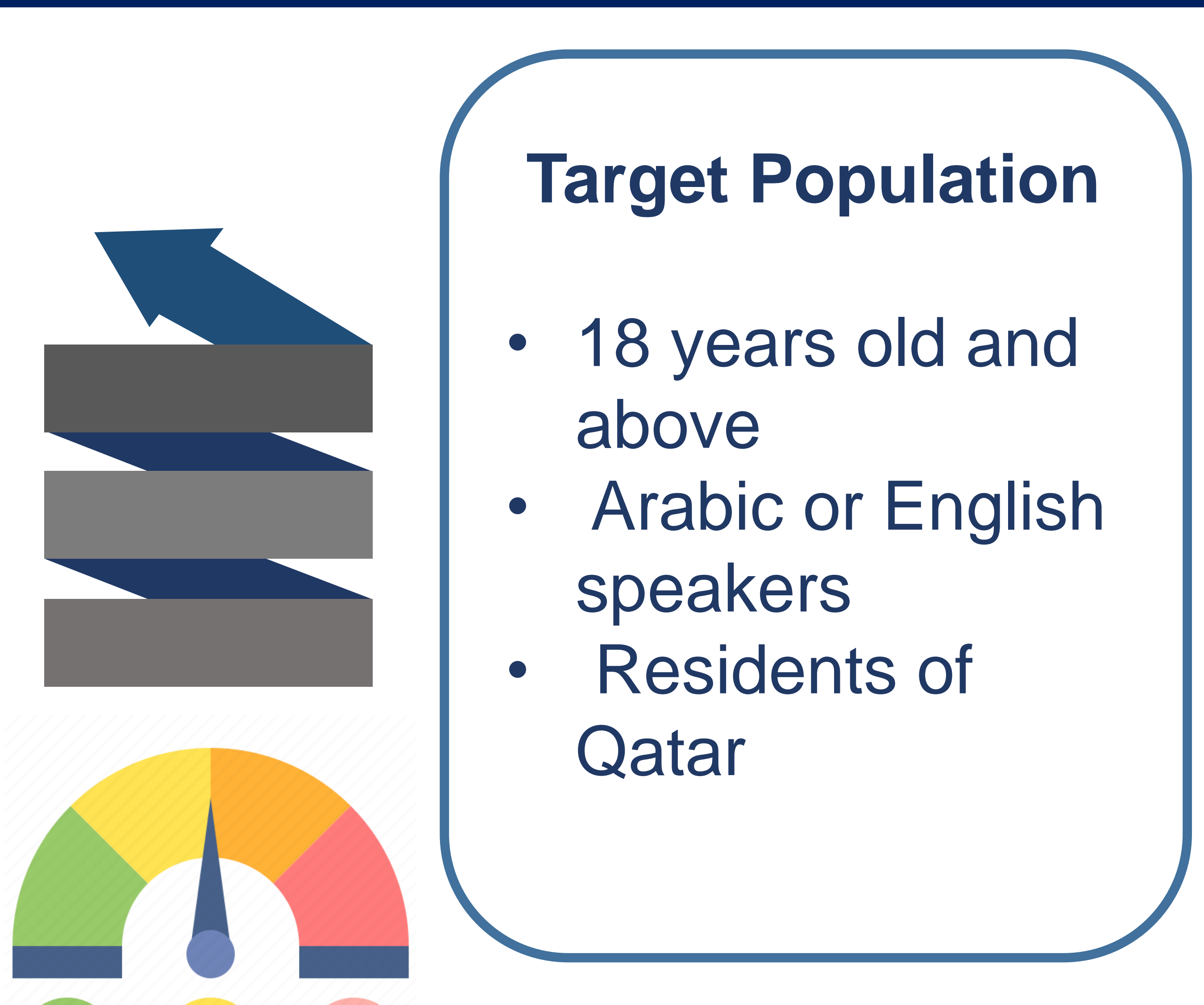

- Sociodemographic characteristics: age, gender, nationality, education, marital status and employment status.

- Pandemic-related questions: COVID-19 related health status, quarantine, familial/social infection or death. Worries or fears related to the using social media to look for coronavirus updates

- Mental health: PHQ-9, GAD-7,PHQ-ADS,UCLA loneliness scale,5-item DUREL religiosity scale, past psychiatric history

\section{Results}

\section{Figure 1: Sample characteristics}

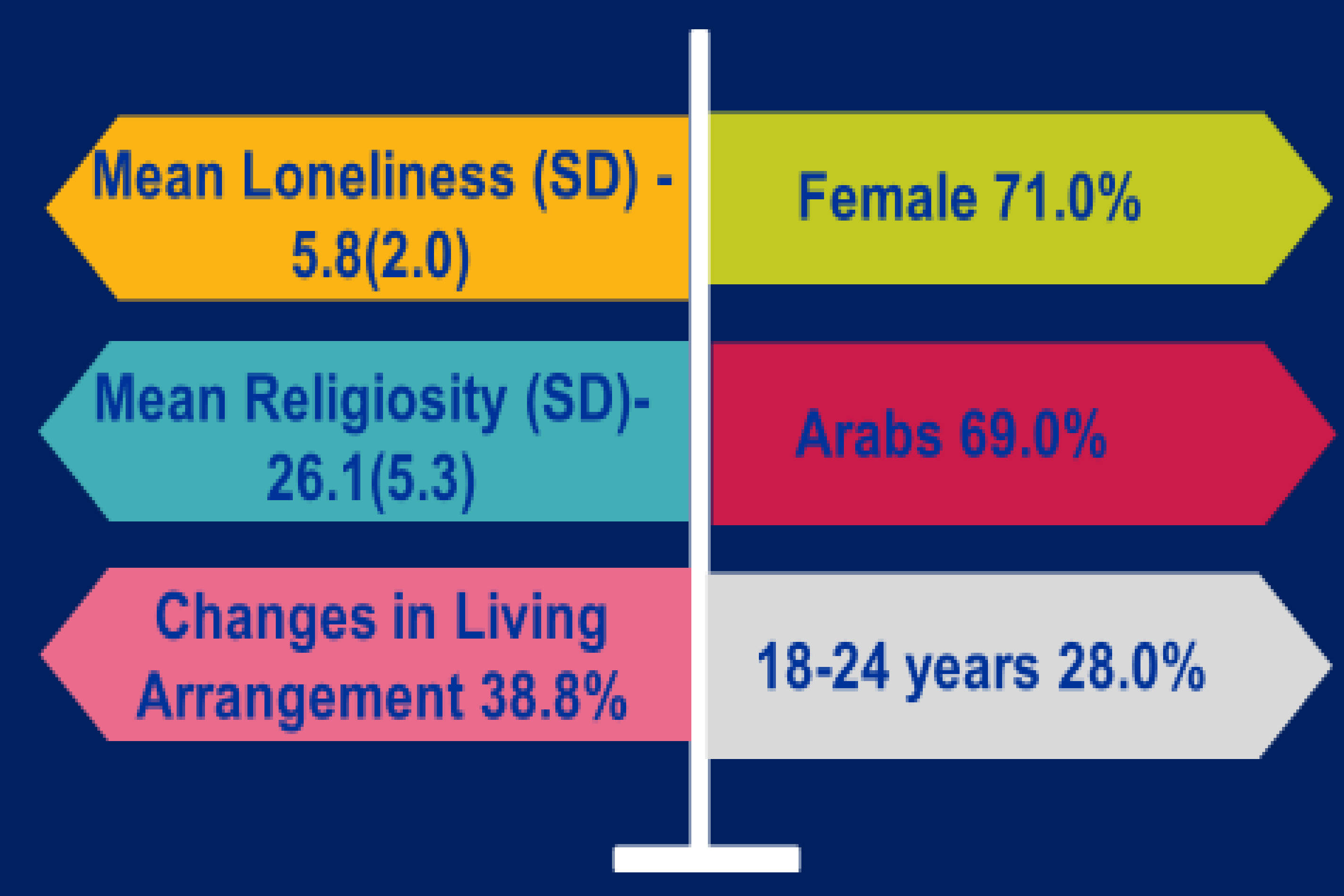

\section{Figure 2: Association with Depression and/ or Anxiety}

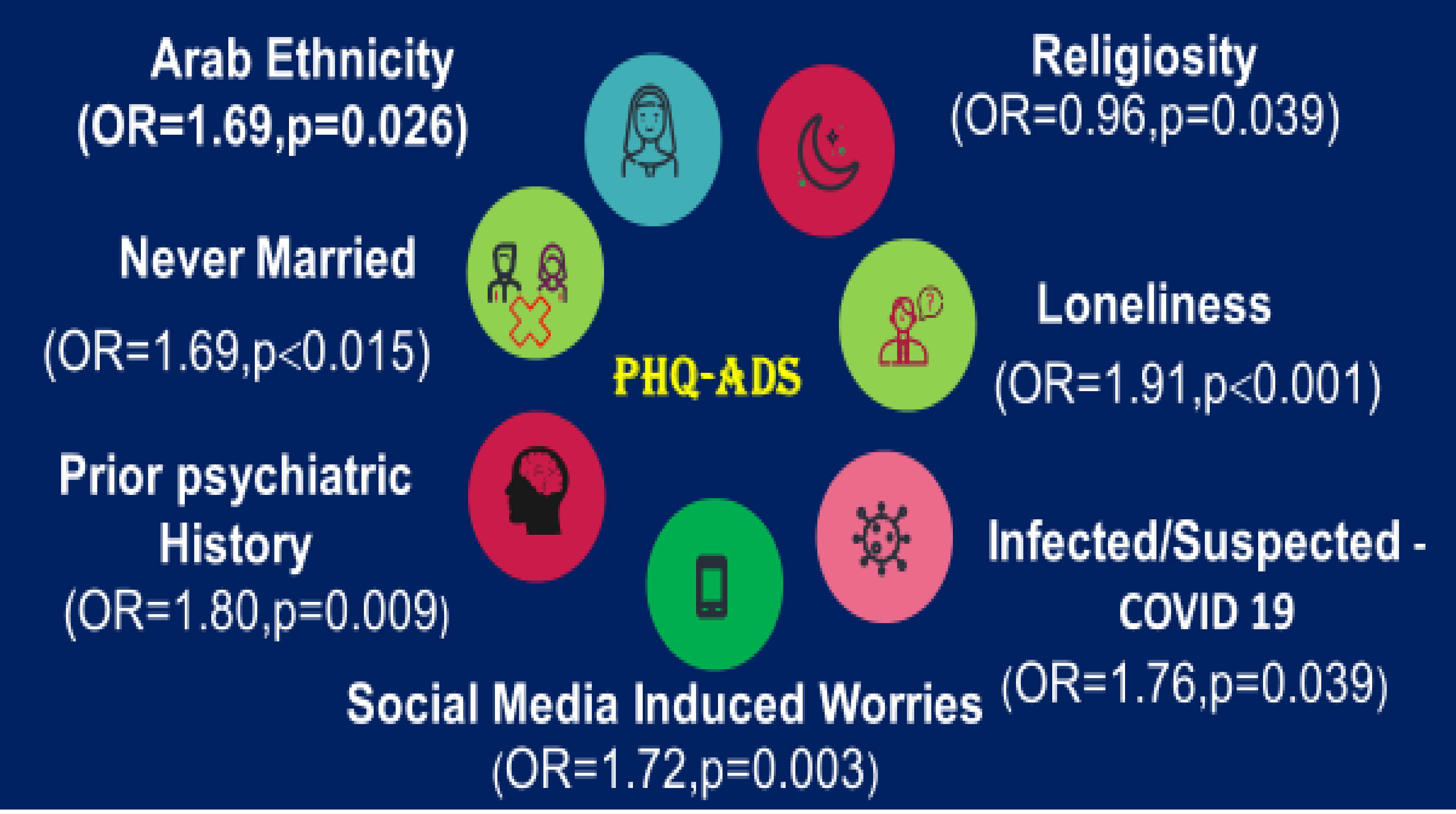

\section{Conclusions}

- The point prevalence of moderate-to-severe depressive or anxiety symptoms was $36.2 \%(95 \% \mathrm{Cl} 33.2-39.4)$.

- The factors we identified may assist in designing support and interventions for those at greater risk of depression and anxiety in future COVID-19 waves or lockdowns in Qatar and other Arab-speaking countries.

\section{Acknowledgements}

